\title{
Nonlocal KdV equations
}

\author{
Metin Gürses ${ }^{\mathrm{a}, 1}$, Aslı Pekcan ${ }^{\mathrm{b}, *, 1}$ \\ a Department of Mathematics, Faculty of Science, Bilkent University, 06800 Ankara, Turkey \\ b Department of Mathematics, Faculty of Science, Hacettepe University, 06800 Ankara, Turkey
}

\section{A R T I C L E I N F O}

\section{Article history:}

Received 23 April 2020

Received in revised form 10 August 2020

Accepted 14 September 2020

Available online 18 September 2020

Communicated by B. Malomed

\begin{abstract}
A B S T R A C T
Writing the Hirota-Satsuma (HS) system of equations in a symmetrical form we find its local and new nonlocal reductions. It turns out that all reductions of the HS system are Korteweg-de Vries (KdV), complex KdV, and new nonlocal KdV equations. We obtain one-soliton solutions of these KdV equations by using the method of Hirota bilinearization.
\end{abstract}

(c) 2020 Elsevier B.V. All rights reserved.

\section{Keywords:}

Hirota-Satsuma system

Local and nonlocal KdV equations

Ablowitz-Musslimani reductions

Hirota method

\section{Introduction}

After Ablowitz and Musslimani introduced nonlocal type of reductions [1-3] we witnessed several works about finding new integrable nonlocal nonlinear partial differential equations and obtaining different kinds of solutions of these local and nonlocal equations. In particular, most of the works focused on nonlocal nonlinear Schrödinger equations (NLS) [1-14], nonlocal modified Korteweg-de Vries (mKdV) equations [2-4], [8], [15-18], nonlocal sine-Gordon (SG) equations [2-4], [19], and so on [20-33].

Recently, it has been shown that systems admitting nonlocal reductions have discrete symmetry transformations leaving the systems invariant. In [34] we showed that a special case of discrete symmetry transformations are actually the nonlocal reductions of the same systems. The connection between local and nonlocal reductions was given in [35], [36]. Among all the nonlocal equations presented so far in the literature, nonlocal KdV equation is missing. It is not possible to obtain it from the nonlocal reductions of the AKNS system. For this purpose, we use the Hirota-Satsuma (HS) system and its reductions.

The original integrable HS system of equations [37-46] is given by

$$
\begin{aligned}
a p_{t} & =\frac{1}{2} p_{x x x}+3 p p_{x}-6 q q_{x} \\
a q_{t} & =-q_{x x x}-3 p q_{x} .
\end{aligned}
$$

This system has a recursion operator [39], [40], [46], $N$-soliton solutions, and infinitely many conserved quantities [52]. Several methods were applied to find solutions of (1) and (2) such as Hirota bilinear method [37], [43], [47], dressing operator technique [48], Darboux transformation method [49], [50], formally variable separation approach [51], and so on. The above form of the HS system is not appropriate for consistent local and nonlocal reductions. It is more useful to map it to a more symmetrical version. By letting

$$
p=\frac{1}{2}(u+v), \quad q=\gamma(u-v)
$$

with $\gamma^{2}=\frac{1}{4}$, we obtain the symmetrical integrable HS system [53-57]

\footnotetext{
* Corresponding author.

E-mail addresses: gurses@fen.bilkent.edu.tr (M. Gürses), aslipekcan@hacettepe.edu.tr (A. Pekcan).

1 These authors contributed equally to this work.
} 


$$
\begin{aligned}
& a u_{t}=-u_{x x x}+3 v_{x x x}-6 u u_{x}+6 v u_{x}+12 v_{x} u, \\
& a v_{t}=-v_{x x x}+3 u_{x x x}-6 v v_{x}+6 u v_{x}+12 v u_{x},
\end{aligned}
$$

where $a$ is a constant. We can also obtain the recursion operator of the symmetrical HS system from the original one. By the use of this recursion operator it is possible to obtain $(2+1)$-dimensional negative HS system.

It is possible to obtain soliton solutions of the above symmetrical HS system by the Hirota bilinearization method. The bilinearizing transformation and the Hirota bilinear form of the original system (1) and (2) were given in [37]. By using the result of [37] with the transformation (3) and introducing two additional constants $u_{0}$ and $v_{0}$ and by letting $u=u_{0}+2(\ln f)_{x x}+\frac{g}{f}$ and $v=v_{0}+2(\ln f)_{x x}-\frac{g}{f}$, we obtain the Hirota bilinear form of the system (4) and (5) as

$$
\begin{aligned}
& \left(a D_{t}+4 D_{x}^{3}+\left(18 u_{0}-6 v_{0}\right) D_{x}\right)\{g \cdot f\}=0, \\
& \left(a D_{x} D_{t}-2 D_{x}^{4}-6\left(u_{0}+v_{0}\right) D_{x}\right)\{f \cdot f\}=-12 g^{2} .
\end{aligned}
$$

If the local or nonlocal reductions of integrable systems are done consistently the reduced equations must also be integrable. In this work we find the integrable reductions of the HS system (4) and (5). All the reductions of the HS system gives a kind of KdV equation. We find the standard KdV equation

$$
a u_{t}=2 u_{x x x}+12 u u_{x}
$$

from the real reduction, complex KdV equation

$$
a u_{t}=-u_{x x x}+3 \bar{u}_{x x x}-6 u u_{x}+6 \bar{u} u_{x}+12 u \bar{u}_{x}
$$

from the complex reduction, nonlocal KdV equation

$$
a u_{t}(x, t)=-u_{x x x}(x, t)+3 u_{x x x}(-x,-t)-6 u(x, t) u_{x}(x, t)+6 u(-x,-t) u_{x}(x, t)+12 u_{x}(-x,-t) u(x, t)
$$

from the real nonlocal reduction, and complex nonlocal KdV equations

$$
a u_{t}(x, t)=-u_{x x x}(x, t)+3 u_{x x x}\left(\varepsilon_{1} x, \varepsilon_{2} t\right)-6 u(x, t) u_{x}(x, t)+6 u\left(\varepsilon_{1} x, \varepsilon_{2} t\right) u_{x}(x, t)+12 u_{x}\left(\varepsilon_{1} x, \varepsilon_{2} t\right) u(x, t)
$$

from the nonlocal complex reductions, where $\varepsilon_{1}^{2}=\varepsilon_{2}^{2}=1$. We give the derivation of all these equations in Section 3 . In Section 4 we obtain the one-soliton solutions of the above local and nonlocal KdV equations by using the one-soliton solutions of the HS system obtained in Section 2 and the reduction constraints.

\section{One-soliton solutions of the HS system}

To obtain the soliton solutions of the reduced equations we need the soliton solutions of the HS system. For this purpose, we use the Hirota bilinear equations given in (6) and (7).

Let $g=\varepsilon g_{1}$ and $f=1+\varepsilon^{2} f_{2}$ where $g_{1}=c e^{\theta_{1}}$ for $\theta_{1}=k_{1} x+\omega_{1} t+\delta_{1}$ and $c$ is a constant, in the Hirota bilinear equations (6) and (7). From the coefficient of $\varepsilon$, we get the dispersion relation

$$
\omega_{1}=\frac{-4 k_{1}^{3}+\left(6 v_{0}-18 u_{0}\right) k_{1}}{a}
$$

if $c \neq 0$. The coefficient of $\varepsilon^{2}$ gives

$$
a f_{2, x t}-2 f_{2, x x x x}-6\left(u_{0}+v_{0}\right) f_{2, x x}=-6 c^{2} e^{2 k_{1} x+2 \omega_{1}+2 \delta_{1}}
$$

yielding

$$
f_{2}=A_{1} e^{k_{2} x+\omega_{2} t+\delta_{2}}+A_{2} e^{2 k_{1} x-\frac{8 k_{1}^{3}}{a} t+2 \delta_{1}},
$$

where

$$
\omega_{2}=\frac{2 k_{2}^{3}+6\left(u_{0}+v_{0}\right) k_{2}}{a},
$$

and

$$
A_{2}=\frac{c^{2}}{8 k_{1}^{2}\left(k_{1}^{2}+2 u_{0}\right)},
$$

and $A_{1}$ is an arbitrary constant. For $c \neq 0, A_{1} \neq 0$, the coefficients of $\varepsilon^{3}$ and $\varepsilon^{4}$ vanish if

$$
k_{2}=k_{1} \pm \sqrt{-k_{1}^{2}-4 u_{0}}
$$

Take $\varepsilon=1$. Hence one-soliton solution of the HS system (4) and (5) is given by the pair $(u(x, t), v(x, t))$, 


$$
\begin{aligned}
& u(x, t)=u_{0}+\frac{c\left(e^{\theta_{1}}+A_{2} e^{3 \theta_{1}}+A_{1} e^{\theta_{1}+\theta_{2}}\right)+8 k_{1}^{2} A_{2} e^{2 \theta_{1}}+2 A_{1} k_{2}^{2} e^{\theta_{2}}+2 A_{1} A_{2}\left(2 k_{1}-k_{2}\right)^{2} e^{\theta_{2}+2 \theta_{1}}}{\left(1+A_{1} e^{\theta_{2}}+A_{2} e^{2 \theta_{1}}\right)^{2}}, \\
& v(x, t)=v_{0}+\frac{-c\left(e^{\theta_{1}}+A_{2} e^{3 \theta_{1}}+A_{1} e^{\theta_{1}+\theta_{2}}\right)+8 k_{1}^{2} A_{2} e^{2 \theta_{1}}+2 A_{1} k_{2}^{2} e^{\theta_{2}}+2 A_{1} A_{2}\left(2 k_{1}-k_{2}\right)^{2} e^{\theta_{2}+2 \theta_{1}}}{\left(1+A_{1} e^{\theta_{2}}+A_{2} e^{2 \theta_{1}}\right)^{2}},
\end{aligned}
$$

where $\theta_{1}=k_{1} x+\omega_{1} t+\delta_{1}, \theta_{2}=k_{2} x+\omega_{2} t+\delta_{2}$ with the dispersion relations (12) and (15), and $A_{2}$ is given by (16). Here $A_{1}, a, c, k_{1}, \delta_{1}, \delta_{2}$ are arbitrary constants and $k_{2}=k_{1} \pm \sqrt{-k_{1}^{2}-4 u_{0}}$. For certain choices of the parameters the functions $u$ and $v$ can develop singularities for finite time. These singularities will be transferred to the local and nonlocal reductions of the HS system.

\section{Reductions of the HS system}

For the HS system (4) and (5) we have two local and two nonlocal reductions.

\section{(a) Local reductions:}

(i) Real reduction: $v(x, t)=k u(x, t), k$ is a real constant.

The system (4) and (5) consistently reduces to the well-known KdV equation

$$
a u_{t}=2 u_{x x x}+12 u u_{x} .
$$

(ii) Complex reduction: $v(x, t)=k \bar{u}(x, t), k$ is a real constant.

Under this reduction the system (4) and (5) reduces to the complex KdV equation

$$
a u_{t}=-u_{x x x}+3 \bar{u}_{x x x}-6 u u_{x}+6 \bar{u} u_{x}+12 u \bar{u}_{x},
$$

consistently, if $a=\bar{a}$.

(b) Nonlocal reductions:

(i) Real nonlocal reductions: $v(x, t)=k u\left(\varepsilon_{1} x, \varepsilon_{2} t\right), \varepsilon_{1}^{2}=\varepsilon_{2}^{2}=1, k$ is a real constant.

When we apply this reduction to the system (4) and (5), it yields that to have a consistent reduction we must have $k=1$ and $\varepsilon_{1} \varepsilon_{2}=1$ i.e. $\varepsilon_{1}=\varepsilon_{2}=-1$. Hence we get the reduced nonlocal ST-reversal KdV equation

$$
a u_{t}(x, t)=-u_{x x x}(x, t)+3 u_{x x x}(-x,-t)-6 u(x, t) u_{x}(x, t)+6 u(-x,-t) u_{x}(x, t)+12 u_{x}(-x,-t) u(x, t) .
$$

(ii) Complex nonlocal reductions: $v(x, t)=k \bar{u}\left(\varepsilon_{1} x, \varepsilon_{2} t\right), \varepsilon_{1}^{2}=\varepsilon_{2}^{2}=1, k$ is a real constant.

This reduction reduces the system (4) and (5) consistently if $k=1$ and $a=\bar{a} \varepsilon_{1} \varepsilon_{2}$. Therefore we have the following nonlocal complex KdV equations:

$$
a u_{t}(x, t)=-u_{x x x}(x, t)+3 \bar{u}_{x x x}\left(\varepsilon_{1} x, \varepsilon_{2} t\right)-6 u(x, t) u_{x}(x, t)+6 \bar{u}\left(\varepsilon_{1} x, \varepsilon_{2} t\right) u_{x}(x, t)+12 \bar{u}_{x}\left(\varepsilon_{1} x, \varepsilon_{2} t\right) u(x, t) .
$$

Explicitly, here we have three nonlocal complex KdV equations.

(a) S-reversal nonlocal complex KdV equation:

$$
a u_{t}(x, t)=-u_{x x x}(x, t)+3 \bar{u}_{x x x}(-x, t)-6 u(x, t) u_{x}(x, t)+6 \bar{u}(-x, t) u_{x}(x, t)+12 \bar{u}_{x}(-x, t) u(x, t),
$$

where $a$ is a pure imaginary number.

\section{(b) T-reversal nonlocal complex KdV equation:}

$$
a u_{t}(x, t)=-u_{x x x}(x, t)+3 \bar{u}_{x x x}(x,-t)-6 u(x, t) u_{x}(x, t)+6 \bar{u}(x,-t) u_{x}(x, t)+12 \bar{u}_{x}(x,-t) u(x, t),
$$

where $a$ is a pure imaginary number.

\section{(c) ST-reversal nonlocal complex KdV equation:}

$$
a u_{t}(x, t)=-u_{x x x}(x, t)+3 \bar{u}_{x x x}(-x,-t)-6 u(x, t) u_{x}(x, t)+6 \bar{u}(-x,-t) u_{x}(x, t)+12 \bar{u}_{x}(-x,-t) u(x, t),
$$

where $a$ is a real number.

\section{One-soliton solutions of the reduced KdV equations}

Using the soliton solutions of the HS system obtained in Section 2 and the reduction formulas we obtain one-soliton solutions of the reduced equations. 


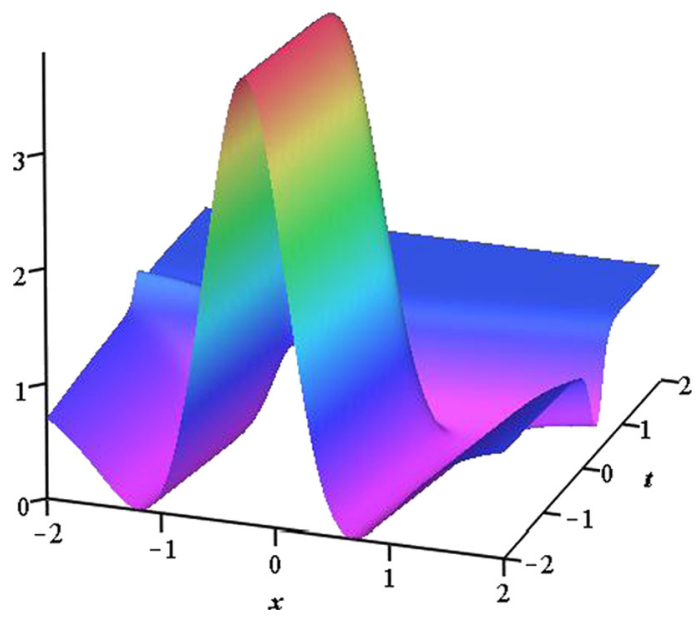

Fig. 1. One-soliton solution for (21) with the parameters $k_{1}=\frac{1}{2}, u_{0}=-1, \alpha=a=A_{1}=1, \delta_{1}=\delta_{2}=0$.

\subsection{One-soliton solutions of the reduced local KdV equations}

(i) $v(x, t)=u(x, t)(\mathrm{KdV}$ equation).

This reduction relation holds if $u_{0}=v_{0}$ and $c=0$ yielding $A_{2}=0$ and $g(x, t)=0$. Hence we obtain the one-soliton solution of the equation (20) as

$$
u(x, t)=u_{0}+\frac{2 A_{1} k_{2}^{2} e^{\theta_{2}}}{\left(1+A_{1} e^{\theta_{2}}\right)^{2}},
$$

where $\theta_{2}=k_{2} x+\frac{\left(2 k_{2}^{3}+12 u_{0} k_{2}\right)}{a} t+\delta_{2}$ and $A_{1}=e^{\delta}$. Here $k_{2}, a, u_{0}, \delta_{2}, \delta$ are some arbitrary constants. Let us take all the parameters real. Then the above solution is nonsingular and bounded soliton solution for $A_{1}>0$. If $A_{1}<0$, the real-valued solution is singular.

(ii) $v(x, t)=\bar{u}(x, t), a=\bar{a}$ (Complex KdV equation).

Let $-k_{1}^{2}-4 u_{0} \geq 0$ i.e. $u_{0} \leq-\frac{k_{1}^{2}}{4}, k_{1}, u_{0} \in \mathbb{R}$ yielding $k_{2} \in \mathbb{R}$. If we use the Type 1 approach [7], [8], [15] we get the following constraints on the parameters of the one-soliton solution of the reduced complex local KdV equation (21):

$$
\begin{aligned}
& \text { 1) } v_{0}=u_{0}, \quad c=-\bar{c}, \quad e^{\delta_{1}}=e^{\bar{\delta}_{1}}, \quad e^{\delta_{2}}=e^{\bar{\delta}_{2}}, \quad A_{1}=\bar{A}_{1}, \\
& \text { 2) } v_{0}=u_{0}, \quad c=\bar{c}, \quad e^{\delta_{1}}=-e^{\bar{\delta}_{1}}, \quad e^{\delta_{2}}=e^{\bar{\delta}_{2}}, \quad A_{1}=\bar{A}_{1} \text {, } \\
& \text { 3) } v_{0}=u_{0}, \quad c=\bar{c}, \quad e^{\delta_{1}}=-e^{\bar{\delta}_{1}}, \quad e^{\delta_{2}}=-e^{\bar{\delta}_{2}}, \quad A_{1}=-\bar{A}_{1} \text {. }
\end{aligned}
$$

The above sets of the constraints give similar solutions. Let us consider the first set of the constraints. Let $c=i \alpha, \alpha \in \mathbb{R}$. Hence one-soliton solution of (21) is

$$
u(x, t)=u_{0}+\frac{i \alpha\left[e^{\theta_{1}}+A_{1} e^{\theta_{1}+\theta_{2}}-\frac{\alpha^{2}}{8 k_{1}^{2}\left(k_{1}^{2}+2 u_{0}\right)} e^{3 \theta_{1}}\right]+2 A_{1} k_{2}^{2} e^{\theta_{2}}-\frac{\alpha^{2}}{k_{1}^{2}+2 u_{0}} e^{2 \theta_{1}}-\frac{A_{1} \alpha^{2}}{4 k_{1}^{2}\left(k_{1}^{2}+2 u_{0}\right)} e^{\theta_{2}+2 \theta_{1}}}{\left(1+A_{1} e^{\theta_{2}}-\frac{\alpha^{2}}{8 k_{1}^{2}\left(k_{1}^{2}+2 u_{0}\right)} e^{2 \theta_{1}}\right)^{2}},
$$

where $\theta_{1}=k_{1} x+\frac{-4 k_{1}^{3}-12 u_{0} k_{1}}{a} t+\delta_{1}, \theta_{2}=k_{2} x+\frac{2 k_{2}^{3}+12 u_{0} k_{2}}{a} t+\delta_{2}$ with $k_{2}=k_{1} \pm \sqrt{-k_{1}^{2}-4 u_{0}}$. For $u_{0}<-\frac{k_{1}^{2}}{2} \leq-\frac{k_{1}^{2}}{4}$ with $A_{1}>0$, the above solution is nonsingular and bounded. When these conditions are relaxed the solution (31) may develop singularity in a finite time.

Example 1. Let us take the parameters as $k_{1}=\frac{1}{2}, u_{0}=-1, \alpha=a=A_{1}=1, \delta_{1}=\delta_{2}=0$. Hence one-soliton solution of (21) becomes

$$
|u(x, t)|^{2}=\frac{H_{1}(x, t)}{\left(1+e^{\left(\frac{1+\sqrt{15}}{2}\right) x+4 t}+\frac{2}{7} e^{x+11 t}\right)^{4}},
$$

where

$$
\begin{aligned}
& H_{1}(x, t)=1+e^{x+11 t}+\frac{36}{49} e^{2 x+22 t}+\frac{4}{49} e^{3 x+33 t}+\frac{16}{2401} e^{4 x+44 t}+\frac{8}{49} e^{(3+\sqrt{15}) x+(33+3 \sqrt{15}) t} \\
& +2 e^{\left(\frac{3+\sqrt{15}}{2}\right) x+\left(\frac{33-3 \sqrt{15}}{2}\right) t}+e^{(2+\sqrt{15}) x+(22-3 \sqrt{15}) t}-(2 \sqrt{15}+12) e^{\left(\frac{3+3 \sqrt{15}}{2}\right) x+\left(\frac{33-9 \sqrt{15}}{2}\right) t} \\
& -(2 \sqrt{15}+12) e^{\left(\frac{1+\sqrt{15}}{2}\right) x+\left(\frac{11-2 \sqrt{15}}{2}\right) t}+(12 \sqrt{15}+53) e^{(1+\sqrt{15}) x+(11-3 \sqrt{15}) t}-\left(\frac{8 \sqrt{15}+20}{49}\right) e^{\left(\frac{5+\sqrt{15}}{2}\right) x+\left(\frac{55+3 \sqrt{15}}{2}\right) t} .
\end{aligned}
$$

The above solution is nonsingular and bounded. The graph of this solution is given in Fig. 1 . 


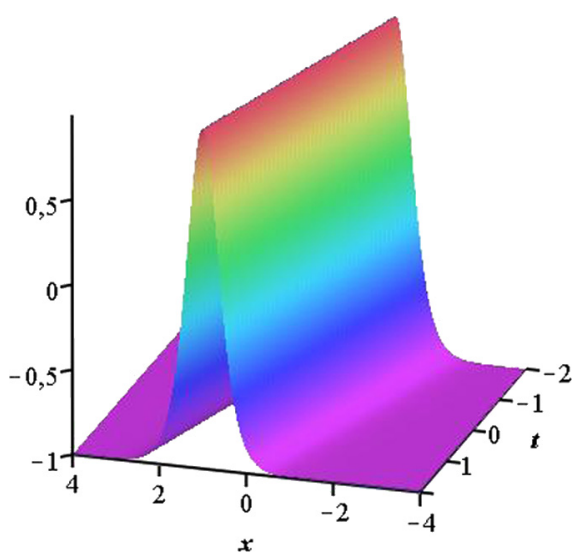

Fig. 2. One-soliton solution for (22) with the parameters $k_{2}=2, u_{0}=-1, a=8$.

4.2. One-soliton solutions of the reduced nonlocal KdV equations

(i) $v(x, t)=u\left(\varepsilon_{1} x, \varepsilon_{2} t\right), \varepsilon_{1}^{2}=\varepsilon_{2}^{2}=1$ (Nonlocal KdV equation).

Recall that the system (4) and (5) reduces to (22) consistently by this reduction if $\varepsilon_{1}=\varepsilon_{2}=-1$. When we consider the reduction with the solutions $u(x, t)$ and $v(x, t)$ with Type 1 approach, we obtain that $k_{1}=k_{2}=0$ giving the trivial solution $u(x, t)=u_{0}$. Therefore we apply Type 2 [8], [15]. In this case we have two possibilities:

$$
\begin{aligned}
& \text { 1) } A_{1}=0, u_{0}=v_{0}, e^{2 \delta_{1}} A_{2}=-1, \\
& \text { 2) } c=0, u_{0}=v_{0}, A_{1} e^{\delta_{2}}= \pm 1 .
\end{aligned}
$$

Take also all the parameters as real numbers and consider the following particular cases.

(i).1) From the first set of the constraints we obtain the one-soliton solution of the reduced nonlocal equation (22) as

$$
u(x, t)=u_{0}+\frac{c\left(e^{k_{1} x+\omega_{1} t+\delta_{1}}-e^{3 k_{1} x+3 \omega_{1} t+\delta_{1}}\right)-8 k_{1}^{2} e^{2 k_{1} x+2 \omega_{1} t}}{\left(1-e^{2 k_{1} x+2 \omega_{1} t}\right)^{2}},
$$

where $e^{2 \delta_{1}}=\frac{-8 k_{1}^{2}\left(k_{1}^{2}+2 u_{0}\right)}{c^{2}}$. This solution is a singular solution on the line $k_{1} x+\omega_{1} t=0$.

(i).2) In the second case we have $A_{2}=0$ and the one-soliton solution of the equation (22) is obtained as

$$
u(x, t)=u_{0}+\sigma_{1} \frac{2 k_{2}^{2} e^{k_{2} x+\omega_{2} t}}{\left(1+\sigma_{1} e^{k_{2} x+\omega_{2} t}\right)^{2}}, \quad \sigma_{1}= \pm 1 .
$$

Let $\sigma_{1}=1, k_{2}, u_{0}, a \in \mathbb{R}$. Then the above solution can be rewritten as

$$
u(x, t)=u_{0}+\frac{k_{2}^{2}}{2} \operatorname{sech}^{2}\left(k_{2} x+\omega_{2} t\right) .
$$

This real-valued solution is nonsingular and bounded. The solution (37) is singular if $\sigma_{1}=-1$.

Example 2. Let us choose the parameters as $k_{1}=2, u_{0}=-1$ giving $k_{2}=2, A_{1}=1, \delta_{2}=0$ satisfying $A_{1} e^{\delta_{2}}=1$, and $a=8$. Hence a nonsingular and bounded one-soliton solution of $(22)$ is

$$
u(x, t)=-1+2 \operatorname{sech}^{2}(2 x-t) .
$$

The graph of the above solution is given in Fig. 2.

(ii) $v(x, t)=\bar{u}\left(\varepsilon_{1} x, \varepsilon_{2} t\right), a=\bar{a} \varepsilon_{1} \varepsilon_{2}, \varepsilon_{1}^{2}=\varepsilon_{2}^{2}=1$ (Nonlocal complex KdV equations).

If we use Type 1 , we obtain the below constraints that must be satisfied by the parameters of the one-soliton solution of the nonlocal reduced complex KdV equations (23),

$$
k_{1}=\varepsilon_{1} \bar{k}_{1}, \quad v_{0}=\bar{u}_{0}=u_{0}, \quad a=\bar{a} \varepsilon_{1} \varepsilon_{2}
$$

with the following possibilities:

$$
\begin{aligned}
& \text { 1) } c=-\bar{c}, A_{1}=\bar{A}_{1}, e^{\delta_{1}}=e^{\bar{\delta}_{1}}, e^{\delta_{2}}=e^{\bar{\delta}_{2}}, \\
& \text { 2) } c=\bar{c}, A_{1}=\bar{A}_{1}, e^{\delta_{1}}=-e^{\bar{\delta}_{1}}, e^{\delta_{2}}=e^{\bar{\delta}_{2}}, \\
& \text { 3) } c=\bar{c}, A_{1}=-\bar{A}_{1}, e^{\delta_{1}}=-e^{\bar{\delta}_{1}}, e^{\delta_{2}}=-e^{\bar{\delta}_{2}},
\end{aligned}
$$


yielding $A_{2}=\bar{A}_{2}, \omega_{1}=\varepsilon_{2} \bar{\omega}_{1}, \omega_{2}=\varepsilon_{2} \bar{\omega}_{2}$, and $k_{2}=\varepsilon_{1} \bar{k}_{2}$ for

$$
u_{0} \leq-\frac{k_{1}^{2}}{4} \text {. }
$$

Here for finding one-soliton solutions of (23) we pick the constraints (40) with (41). We have three types of nonlocal reduced complex equations and we will consider one-soliton solutions of them separately. Note that we will mainly focus on some particular cases giving nonsingular and bounded solutions.

\section{(a) S-reversal nonlocal complex KdV equation:}

In this case, according to the constraints (40) and (41) with $a=\bar{a} \varepsilon_{1} \varepsilon_{2}$ and (44), since $\left(\varepsilon_{1}, \varepsilon_{2}\right)=(-1,1)$ we have $k_{1}=-\bar{k}_{1}$ and $a=-\bar{a}$. Let $k_{1}=i \alpha_{1}, k_{2}=i \alpha_{1}\left(1 \pm \sqrt{-1+\frac{4 u_{0}}{\alpha_{1}^{2}}}\right)=i \alpha_{2}, c=i \alpha_{3}, a=i \alpha_{4}$ for $\alpha_{j}, j=1,2,3,4 \in \mathbb{R}$. Hence we obtain the one-soliton solution of the S-reversal nonlocal complex equation (24) as

$$
u(x, t)=u_{0}+\frac{B_{1}(x, t)}{\left(B_{2}(x, t)\right)^{2}},
$$

where

$$
\begin{aligned}
& B_{1}(x, t)=i \alpha_{3}\left(e^{i \alpha_{1} x+\omega_{1} t+\delta_{1}}+A_{1} e^{i\left(\alpha_{1}+\alpha_{2}\right) x+\left(\omega_{1}+\omega_{2}\right) t+\delta_{1}+\delta_{2}}+A_{2} e^{3 i \alpha_{1} x+3 \omega_{1} t+3 \delta_{1}}\right) \\
& -2 \alpha_{2}^{2} A_{1} e^{i \alpha_{2} x+\omega_{2} t+\delta_{2}}-8 \alpha_{1}^{2} A_{2} e^{2 i \alpha_{1} x+2 \omega_{1} t+2 \delta_{1}}-2 A_{1} A_{2}\left(2 \alpha_{1}-\alpha_{2}\right)^{2} e^{i\left(2 \alpha_{1}+\alpha_{2}\right) x+\left(2 \omega_{1}+\omega_{2}\right) t+2 \delta_{1}+\delta_{2}}
\end{aligned}
$$

and

$$
B_{2}(x, t)=1+A_{1} e^{i \alpha_{2} x+\omega_{2} t+\delta_{2}}+A_{2} e^{2 i \alpha_{1} x+2 \omega_{1} t+2 \delta_{1}},
$$

where

$$
\omega_{1}=\frac{4 \alpha_{1}^{3}-12 u_{0} \alpha_{1}}{\alpha_{4}}, \omega_{2}=\frac{-2 \alpha_{2}^{3}+12 u_{0} \alpha_{2}}{\alpha_{4}}, A_{2}=\frac{\alpha_{3}^{2}}{8 \alpha_{1}^{2}\left(2 u_{0}-\alpha_{1}\right)} .
$$

If we consider the real-valued solution we get

$$
|u(x, t)|^{2}=\frac{H_{1}(x, t)}{\left(H_{2}(x, t)\right)^{2}},
$$

where

$$
\begin{aligned}
& H_{2}(x, t)=1+2 A_{1} e^{\omega_{2} t+\delta_{2}} \cos \left(\alpha_{2} x\right)+2 A_{2} e^{2 \omega_{1} t+2 \delta_{1}} \cos \left(2 \alpha_{1} x\right)+A_{1}^{2} e^{2 \omega_{2} t+2 \delta_{2}}+A_{2}^{2} e^{4 \omega_{1} t+4 \delta_{1}} \\
& +2 A_{1} A_{2} e^{\left(2 \omega_{1}+\omega_{2}\right) t+2 \delta_{1}+\delta_{2}} \cos \left(\left(2 \alpha_{1}-\alpha_{2}\right) x\right) .
\end{aligned}
$$

Here $H_{1}(x, t)$ is a huge expression so we are not giving it here explicitly. We will consider some particular cases.

(a).1) Let us consider the case when $A_{1}=0, u_{0} \neq \frac{\alpha_{1}^{2}}{3} \geq \frac{\alpha_{1}^{2}}{4}$, and $\alpha_{3} \neq 0$. The function $H_{2}(x, t)$ in the denominator of the solution (49) becomes

$$
H_{2}(x, t)=\left(A_{2} e^{2 \omega_{1} t+2 \delta_{1}}+\cos \left(2 \alpha_{1} x\right)\right)^{2}+\sin ^{2}\left(2 \alpha_{1} x\right) .
$$

Therefore the solution (49) corresponding to this case is singular for $x=\frac{n \pi}{2 \alpha_{1}}$ and $A_{2} e^{2 \omega_{1} t+2 \delta_{1}}+(-1)^{n}=0$, where $n$ is an integer.

Note that in addition to $A_{1}=0$ if we take $u_{0}=\frac{\alpha_{1}^{2}}{3}$ yielding $\omega_{1}=0$, we have

$$
|u(x, t)|^{2}=\frac{H_{1}(x, t)}{4 A_{2}^{2} e^{4 \delta_{1}}\left(B+\cos \left(2 \alpha_{1} x\right)\right)^{2}}, \quad B=\frac{1+A_{2}^{2} e^{4 \delta_{1}}}{2 A_{2} e^{2 \delta_{1}}},
$$

where

$$
\begin{aligned}
& H_{1}(x, t)=\frac{1}{9} \alpha_{1}^{4}\left(A_{2}^{4} e^{8 \delta_{1}}+1\right)+\alpha_{3}^{2} e^{2 \delta_{1}}\left(A_{2}^{2} e^{4 \delta_{1}}+1\right)+\frac{484}{9} \alpha_{1}^{4} A_{2}^{2} e^{4 \delta_{1}}+\frac{2}{9} \alpha_{1}^{4} A_{2}^{2} e^{4 \delta_{1}} \cos \left(4 \alpha_{1} x\right) \\
& +2 A_{2} e^{2 \delta_{1}}\left(\alpha_{3}^{2} e^{2 \delta_{1}}-\frac{22}{9} \alpha_{1}^{4}\left(A_{2}^{2} e^{4 \delta_{1}}+1\right)\right) \cos \left(2 \alpha_{1} x\right)+\frac{2}{3} \alpha_{1}^{2} \alpha_{3} A_{2} e^{3 \delta_{1}}\left(A_{2} e^{2 \delta_{1}}-1\right) \sin \left(3 \alpha_{1} x\right) \\
& +\left(\frac{2}{3} \alpha_{1}^{2} \alpha_{3} e^{\delta_{1}}\left(A_{2}^{3} e^{6 \delta_{1}}-1\right)+\frac{44}{3} \alpha_{1}^{2} \alpha_{3} A_{2} e^{3 \delta_{1}}\left(A_{2} e^{2 \delta_{1}}-1\right)\right) \sin \left(\alpha_{1} x\right) .
\end{aligned}
$$

For $B>1$ or $B<-1$ the solution (52) is nonsingular and bounded solution. Here if $-1 \leq B \leq 1$ the solution (52) is singular.

Example 3. For this case choose the parameters as $\alpha_{1}=\frac{1}{2}, \alpha_{3}=1, \delta_{1}=0$. This gives $A_{2}=-6$. Hence solution of (24) becomes

$$
|u(x, t)|^{2}=\frac{24049+8040 \cos (x)+72 \cos (2 x)+16968 \sin \left(\frac{x}{2}\right)+1008 \sin \left(\frac{3}{2} x\right)}{144(37-12 \cos (x))^{2}} .
$$

This is a nonsingular and bounded periodic wave solution. The graph of the above solution is given in Fig. 3. 


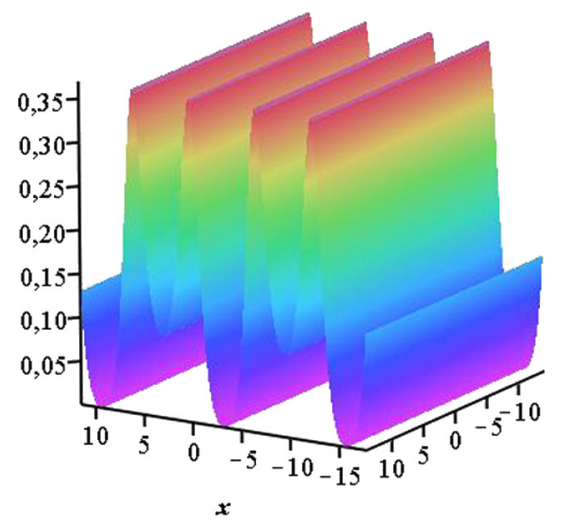

Fig. 3. Periodic wave solution for (24) with the parameters $\alpha_{1}=\frac{1}{2}, \alpha_{3}=1, \delta_{1}=0$.

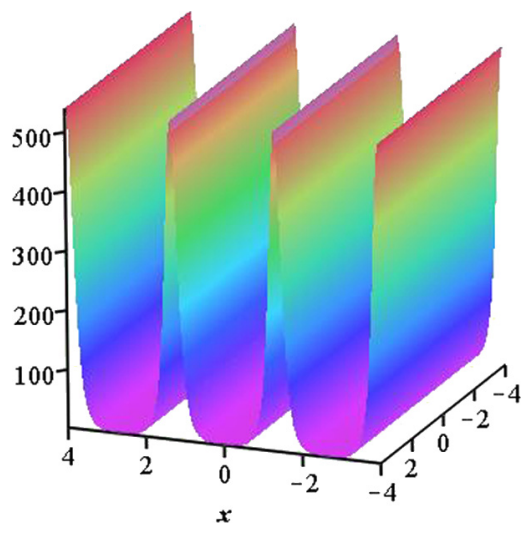

Fig. 4. Periodic wave solution for (24) with the parameters $\alpha_{1}=\frac{1}{2}, A_{1}=2, \delta_{2}=0$.

(a).2) Let us now assume that $\alpha_{3}=0$ i.e. $c=A_{2}=0$, and $u_{0} \neq \frac{\alpha_{2}^{2}}{6}, A_{1} \neq 0$. In this case the function $H_{2}(x, t)$ in the denominator of the solution (49) becomes

$$
H_{2}(x, t)=\left(A_{1} e^{\omega_{2} t+\delta_{2}}+\cos \left(\alpha_{2} x\right)\right)^{2}+\sin ^{2}\left(\alpha_{2} x\right) .
$$

Therefore the solution (49) corresponding to this case is singular for $x=\frac{n \pi}{\alpha_{2}}$ and $A_{1} e^{\omega_{2} t+\delta_{2}}+(-1)^{n}=0$, where $n$ is an integer.

Note that in addition to $\alpha_{3}=0$ if we also take $u_{0}=\frac{\alpha_{2}^{2}}{6} \geq \frac{\alpha_{1}^{2}}{4}$ i.e. $\omega_{2}=0$, the solution (49) turns to be

$$
|u(x, t)|^{2}=\frac{\alpha_{1}^{4}(7 \pm 4 \sqrt{3})\left[1+A_{1}^{4} e^{4 \delta_{2}}+100 A_{1}^{2} e^{2 \delta_{2}}-\left(20 A_{1}^{3} e^{3 \delta_{2}}+20 A_{1} e^{\delta_{2}}\right) \cos \left(\alpha_{1}(3 \pm \sqrt{3}) x\right)+2 A_{1}^{2} e^{2 \delta_{2}} \cos \left(2 \alpha_{1}(3 \pm \sqrt{3}) x\right)\right]}{\left[\left(A_{1} e^{\delta_{2}}+\cos \left(\alpha_{1}(3 \pm \sqrt{3}) x\right)\right)^{2}+\sin ^{2}\left(\alpha_{1}(3 \pm \sqrt{3}) x\right)\right]^{2}} .
$$

The above solution is nonsingular and bounded if $A_{1} e^{\delta_{2}}<-1$ or $A_{1} e^{\delta_{2}}>1$.

Example 4. Take $\alpha_{1}=\frac{1}{2}, \alpha_{2}=\frac{1}{2}(3+\sqrt{3}), A_{1}=2, \delta_{2}=0$. Hence solution of (24) becomes

$$
|u(x, t)|^{2}=\frac{(7+4 \sqrt{3})\left[417-200 \cos \left(\frac{1}{2}(3+\sqrt{3}) x\right)+8 \cos ((3+\sqrt{3}) x)\right]}{16\left[5+4 \cos \left(\frac{1}{2}(3+\sqrt{3}) x\right)\right]^{2}} .
$$

The above solution is a nonsingular and bounded periodic wave solution. The graph of this solution is given in Fig. 4 .

\section{(b) T-reversal nonlocal complex KdV equation:}

According to the constraints (40) and (41) with $a=\bar{a} \varepsilon_{1} \varepsilon_{2}$ and (44), since $\left(\varepsilon_{1}, \varepsilon_{2}\right)=(1,-1)$ here we have $k_{1}=\bar{k}_{1}$ and $a=-\bar{a}$. Let $c=i \alpha_{1}$ and $a=i \alpha_{2}$ for $\alpha_{j}, j=1,2, \in \mathbb{R}$ yielding

$$
\omega_{1}=\frac{i\left(4 k_{1}^{3}+12 u_{0} k_{1}\right)}{\alpha_{2}}=i \alpha_{3}, \omega_{2}=\frac{-i\left(2 k_{1}^{3}+12 u_{0} k_{1}\right)}{\alpha_{2}}=i \alpha_{4}, A_{2}=-\frac{\alpha_{1}^{2}}{8 k_{1}^{2}\left(k_{1}^{2}+2 u_{0}\right)} .
$$

Therefore we obtain the one-soliton solution of the T-reversal nonlocal complex equation (25) as

$$
u(x, t)=u_{0}+\frac{B_{1}(x, t)}{\left(B_{2}(x, t)\right)^{2}},
$$




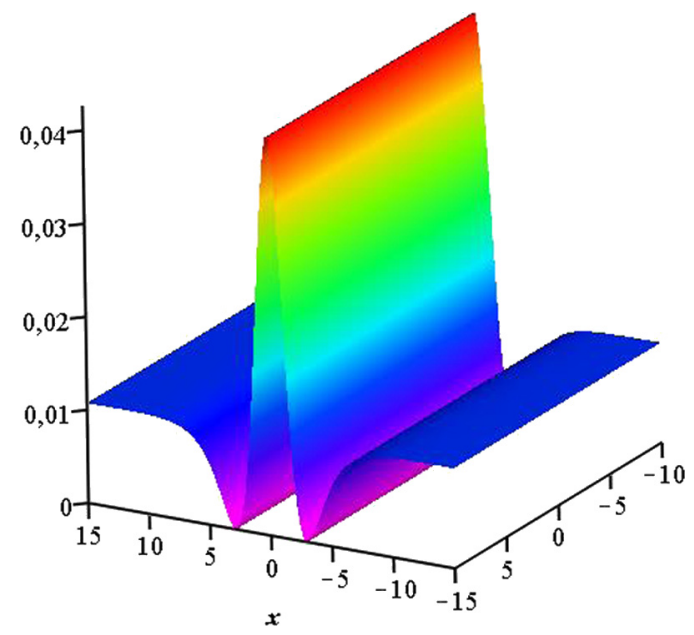

Fig. 5. One-soliton solution for (25) with the parameters $k_{1}=\frac{1}{6}, k_{2}=\frac{1}{6}(3+\sqrt{3}), A_{1}=1, \delta_{2}=0$.

where

$$
\begin{aligned}
& B_{1}(x, t)=i \alpha_{1}\left(e^{k_{1} x+i \alpha_{3} t+\delta_{1}}+A_{1} e^{\left(k_{1}+k_{2}\right) x+i\left(\alpha_{3}+\alpha_{4}\right) t+\delta_{1}+\delta_{2}}+A_{2} e^{3 k_{1} x+3 i \alpha_{3} t+3 \delta_{1}}\right) \\
& +2 k_{2}^{2} A_{1} e^{k_{2} x+i \alpha_{4} t+\delta_{2}}+8 k_{1}^{2} A_{2} e^{2 k_{1} x+2 i \alpha_{3} t+2 \delta_{1}}+2 A_{1} A_{2}\left(2 k_{1}-k_{2}\right)^{2} e^{\left(2 k_{1}+k_{2}\right) x+i\left(2 \alpha_{3}+\alpha_{4}\right) t+2 \delta_{1}+\delta_{2}}
\end{aligned}
$$

and

$$
B_{2}(x, t)=1+A_{1} e^{k_{2} x+i \alpha_{4} t+\delta_{2}}+A_{2} e^{2 k_{1} x+2 i \alpha_{3} t+2 \delta_{1}} .
$$

Let us consider the real-valued solution $|u(x, t)|^{2}$. We have

$$
|u(x, t)|^{2}=\frac{H_{1}(x, t)}{\left(H_{2}(x, t)\right)^{2}},
$$

where

$$
\begin{aligned}
& H_{2}(x, t)=1+2 A_{2} e^{2 k_{1} x+2 \delta_{1}} \cos \left(2 \alpha_{3} t\right)+2 A_{1} e^{k_{2} x+\delta_{2}} \cos \left(\alpha_{4} t\right)+A_{1}^{2} e^{2 k_{2} x+2 \delta_{2}}+A_{2}^{2} e^{4 k_{1} x+4 \delta_{1}} \\
& +2 A_{1} A_{2} e^{\left(2 k_{1}+k_{2}\right) x+2 \delta_{1}+\delta_{2}} \cos \left(\left(2 \alpha_{3}-\alpha_{4}\right) t\right) .
\end{aligned}
$$

Here again $H_{1}(x, t)$ is a huge expression so we are not expressing it here. Let us consider two particular cases.

(b).1) Take $\alpha_{1}=0$ yielding $A_{2}=c=0$, and $u_{0}=-\frac{k_{2}^{2}}{6}$ giving $\alpha_{4}=0$. Note that in this case $k_{2}=(3 \pm \sqrt{3}) k_{1}$. The solution (62) becomes

$$
|u(x, t)|^{2}=\frac{k_{1}^{4}(7 \pm 4 \sqrt{3})\left[1-20 A_{1} e^{(3 \pm \sqrt{3}) k_{1} x+\delta_{2}}+102 A_{1}^{2} e^{2(3 \pm \sqrt{3}) k_{1} x+2 \delta_{2}}-20 A_{1}^{3} e^{3(3 \pm \sqrt{3}) k_{1} x+3 \delta_{2}}+A_{1}^{4} e^{4(3 \pm \sqrt{3}) k_{1} x+4 \delta_{2}}\right]}{\left[A_{1} e^{(3 \pm \sqrt{3}) k_{1} x+\delta_{2}}+1\right]^{4}} .
$$

The above solution is nonsingular and bounded for $A_{1}>0$.

Example 5. Take the parameters as $k_{1}=\frac{1}{6}, k_{2}=\frac{1}{6}(3+\sqrt{3}), A_{1}=1, \delta_{2}=0$. Hence one-soliton solution of (25) becomes

$$
|u(x, t)|^{2}=\frac{(7+4 \sqrt{3})\left[e^{\frac{2}{3}(3+\sqrt{3}) x}-20 e^{\frac{1}{2}(3+\sqrt{3}) x}+102 e^{\frac{1}{3}(3+\sqrt{3}) x}-20 e^{\frac{1}{6}(3+\sqrt{3}) x}\right]}{1296\left(e^{\frac{1}{6}(3+\sqrt{3}) x}+1\right)^{4}} .
$$

The above solution is a nonsingular and bounded solution. The graph of this solution is given in Fig. 5 .

(b).2) Choose now $\alpha_{3}=0$ that is $u_{0}=-\frac{k_{1}^{2}}{3}$ and $\alpha_{1}=0$ yielding $A_{2}=c=0$. In this case $k_{2}=\left(1 \pm \frac{1}{\sqrt{3}}\right) k_{1}$. Take the plus sign for $k_{2}$. The solution (62) becomes

$$
|u(x, t)|^{2}=\frac{H_{1}(x, t)}{9\left[\left(A_{1} e^{\left(1+\frac{1}{\sqrt{3}}\right) k_{1} x+\delta_{2}}+\cos \left(\alpha_{4} t\right)\right)^{2}+\sin ^{2}\left(\alpha_{4} t\right)\right]^{2}},
$$

where

$$
\begin{aligned}
& H_{1}(x, t)=k_{1}^{4}\left[1+A_{1}^{4} e^{4 k_{1}\left(1+\frac{1}{\sqrt{3}}\right) k_{1} x+4 \delta_{2}}+12 A_{1}^{2}(7+4 \sqrt{3}) e^{2 k_{1}\left(1+\frac{1}{\sqrt{3}}\right) k_{1} x+2 \delta_{2}}+2 A_{1}^{2} e^{2 k_{1}\left(1+\frac{1}{\sqrt{3}}\right) k_{1} x+2 \delta_{2}} \cos \left(2 \alpha_{4} t\right)\right. \\
& \left.-4 A_{1}(3+2 \sqrt{3})\left(e^{3 k_{1}\left(1+\frac{1}{\sqrt{3}}\right) k_{1} x+3 \delta_{2}}+A_{1}^{2} e^{k_{1}\left(1+\frac{1}{\sqrt{3}}\right) k_{1} x+\delta_{2}}\right) \cos \left(\alpha_{4} t\right)\right] .
\end{aligned}
$$




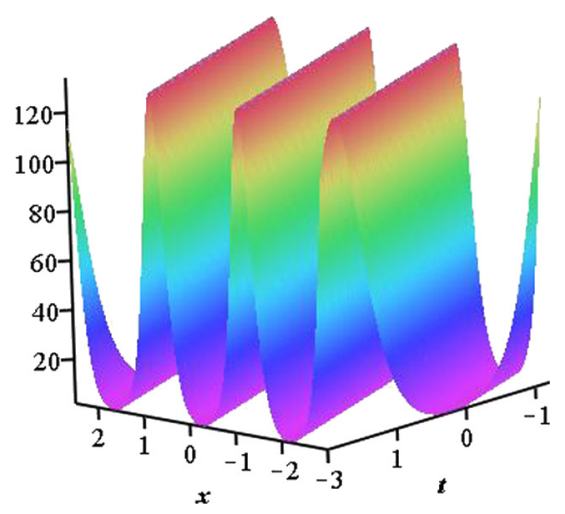

Fig. 6. Periodic wave solution for (26) with the parameters $\alpha_{1}=\frac{1}{2}, u_{0}=2, \delta_{2}=0, A_{1}=-4, a=4$.

Here $\alpha_{4}=-\frac{8 k_{1}^{3} \sqrt{3}}{9 \alpha_{2}}$. The above solution is singular for $A_{1} e^{k_{2} x+\delta_{2}}+(-1)^{n}=0$ at $t=\frac{n \pi}{\alpha_{4}}$, where $n$ is an integer.

(c) ST-reversal nonlocal complex KdV equation:

In this case according to the constraints (40) and (41) with $a=\bar{a} \varepsilon_{1} \varepsilon_{2}$ and (44), since $\left(\varepsilon_{1}, \varepsilon_{2}\right)=(-1,-1)$ we have $k_{1}=-\bar{k}_{1}$ and $a=\bar{a}$. Let $k_{1}=i \alpha_{1}, k_{2}=i \alpha_{1}\left(1 \pm \sqrt{-1+\frac{4 u_{0}}{\alpha_{1}^{2}}}\right)=i \alpha_{2}, c=i \alpha_{3}$ for $\alpha_{j}, j=1,2,3 \in \mathbb{R}$ yielding

$$
\omega_{1}=\frac{i\left(4 \alpha_{1}^{3}-12 u_{0} \alpha_{1}\right)}{a}=i \alpha_{4}, \omega_{2}=\frac{i\left(-2 \alpha_{2}^{3}+12 u_{0} \alpha_{2}\right)}{a}=i \alpha_{5}, A_{2}=\frac{\alpha_{3}^{2}}{8 \alpha_{1}^{2}\left(2 u_{0}-\alpha_{1}^{2}\right)} .
$$

Therefore we obtain the one-soliton solution of the ST-reversal nonlocal complex equation (26) as

$$
u(x, t)=u_{0}+\frac{B_{1}(x, t)}{\left(B_{2}(x, t)\right)^{2}}
$$

where

$$
\begin{aligned}
& B_{1}(x, t)=i \alpha_{3}\left(e^{i \alpha_{1} x+i \alpha_{4} t+\delta_{1}}+A_{1} e^{i\left(\alpha_{1}+\alpha_{2}\right) x+i\left(\alpha_{4}+\alpha_{5}\right) t+\delta_{1}+\delta_{2}}+A_{2} e^{3 i \alpha_{1} x+3 i \alpha_{4} t+3 \delta_{1}}\right) \\
& +2 A_{1} k_{2}^{2} e^{i \alpha_{2} x+i \alpha_{5} t+\delta_{2}}+8 k_{1}^{2} A_{2} e^{2 i \alpha_{1} x+2 i \alpha_{4} t+2 \delta_{1}}-2 A_{1} A_{2}\left(2 \alpha_{1}-\alpha_{2}\right)^{2} e^{i\left(2 \alpha_{1}+\alpha_{2}\right) x+i\left(2 \alpha_{4}+\alpha_{5}\right) t+2 \delta_{1}+\delta_{2}}
\end{aligned}
$$

and

$$
B_{2}(x, t)=1+A_{1} e^{i \alpha_{2} x+i \alpha_{5} t+\delta_{2}}+A_{2} e^{2 i \alpha_{1} x+2 i \alpha_{4} t+2 \delta_{1}} .
$$

When we consider the real-valued solution $|u(x, t)|^{2}$ we get

$$
|u(x, t)|^{2}=\frac{H_{1}(x, t)}{\left(H_{2}(x, t)\right)^{2}},
$$

where

$$
\begin{aligned}
& H_{2}(x, t)=1+2 A_{2} e^{2 \delta_{1}} \cos \left(2 \alpha_{1} x+2 \alpha_{4} t\right)+2 A_{1} e^{\delta_{2}} \cos \left(\alpha_{2} x+\alpha_{5} t\right)+A_{1}^{2} e^{2 \delta_{2}}+A_{2}^{2} e^{4 \delta_{1}} \\
& +2 A_{1} A_{2} e^{2 \delta_{1}+\delta_{2}} \cos \left(\left(2 \alpha_{1}-\alpha_{2}\right) x+\left(2 \alpha_{4}-\alpha_{5}\right) t\right) .
\end{aligned}
$$

Here also $H_{1}(x, t)$ is a huge expression so we are not giving it here explicitly. We will deal with some particular cases.

(c).1) Consider the case when $\alpha_{3}=0$ yielding $A_{2}=0$. Take also $A_{1} \neq 0$. In this case the solution (72) of the nonlocal complex equation (26) becomes

$$
|u(x, t)|^{2}=\frac{4 u_{0} A_{1}\left(u_{0}-\alpha_{2}^{2}\right) e^{\delta_{2}}\left(1+A_{1}^{2} e^{2 \delta_{2}}\right) \cos \left(\alpha_{2} x+\alpha_{5} t\right)+2 u_{0}^{2} A_{1}^{2} e^{2 \delta_{2}} \cos \left(2 \alpha_{2} x+2 \alpha_{5} t\right)+4 A_{1}^{2} e^{2 \delta_{2}}\left(u_{0}-\alpha_{2}^{2}\right)^{2}}{\left[\left(A_{1} e^{\delta_{2}}+\cos \left(\alpha_{2} x+\alpha_{5} t\right)\right)^{2}+\sin ^{2}\left(\alpha_{2} x+\alpha_{5} t\right)\right]^{2}}
$$

This solution is nonsingular and bounded if $A_{1} e^{\delta_{2}}>1$ or $A_{1} e^{\delta_{2}}<-1$. When these conditions are relaxed the solution (74) may develop singularity in a finite time.

Example 6. Take the parameters of the solution (74) as $\alpha_{1}=\frac{1}{2}, u_{0}=2, \delta_{2}=0, A_{1}=-4$, and $a=4$. Hence the solution (74) turns to be

$$
|u(x, t)|^{2}=\frac{1284-64(1+\sqrt{31})^{2}+4(1+\sqrt{31})^{4}+\left(136(1+\sqrt{31})^{2}-1088\right) \cos (\psi)+128 \cos (2 \psi)}{[17-8 \cos (\psi)]^{2}},
$$

where $\psi=\frac{1}{2}(1+\sqrt{31}) x+\left(3(1+\sqrt{31})-\frac{1}{16}(1+\sqrt{31})^{3}\right) t$. This is a nonsingular and bounded periodic wave solution. The graph of the above solution is given in Fig. 6 . 


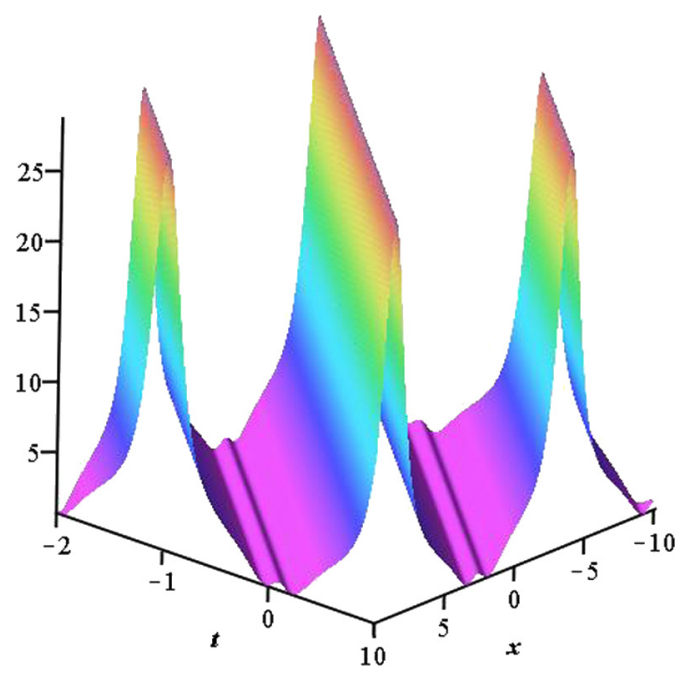

Fig. 7. Periodic wave solution for (26) with the parameters $\alpha_{1}=\frac{1}{2}, u_{0}=2, \delta_{1}=0, \alpha_{3}=5, a=4$.

(c).2) Here we again consider a particular case. Take $A_{1}=0$ and $A_{2} \neq 0$. Therefore the solution (72) of the nonlocal complex equation (26) becomes

$$
|u(x, t)|^{2}=\frac{H_{1}(x, t)}{\left[\left(A_{2} e^{2 \delta_{1}}+\cos \left(2 \alpha_{1} x+2 \alpha_{4} t\right)\right)^{2}+\sin ^{2}\left(2 \alpha_{1} x+2 \alpha_{4} t\right)\right]^{2}},
$$

where

$$
\begin{aligned}
& H_{1}(x, t)=2 \alpha_{3} e^{\delta_{1}}\left[2 u_{0} A_{2} e^{2 \delta_{1}}-u_{0}-2 u_{0} A_{2}^{2} e^{4 \delta_{1}}+u_{0} A_{2}^{3} e^{6 \delta_{1}}-8 A_{2} \alpha_{1}^{2} e^{2 \delta_{1}}+8 A_{2}^{2} \alpha_{1}^{2} e^{4 \delta_{1}}\right] \sin \left(\alpha_{1} x+\alpha_{4} t\right) \\
& +2 e^{2 \delta_{1}} A_{2}\left[2 u_{0}^{2}-8 u_{0} \alpha_{1}^{2}+\alpha_{3}^{2} e^{2 \delta_{1}}+2 u_{0}^{2} A_{2}^{2} e^{4 \delta_{1}}-8 u_{0} A_{2}^{2} \alpha_{1}^{2} e^{4 \delta_{1}}\right] \cos \left(2 \alpha_{1} x+2 \alpha_{4} t\right) \\
& +2 u_{0} \alpha_{3} A_{2} e^{3 \delta_{1}}\left[A_{2} e^{2 \delta_{1}}-1\right] \sin \left(3 \alpha_{1} x+3 \alpha_{4} t\right)+2 u_{0}^{2} A_{2}^{2} e^{4 \delta_{1}} \cos \left(4 \alpha_{1} x+4 \alpha_{4} t\right)+u_{0}^{2} \\
& +e^{2 \delta_{1}}\left[\alpha_{3}^{2}+64 A_{2}^{2} \alpha_{1}^{4} e^{2 \delta_{1}}-32 u_{0} A_{2}^{2} \alpha_{1}^{2} e^{2 \delta_{1}}+4 u_{0}^{2} A_{2}^{2} e^{2 \delta_{1}}+\alpha_{3}^{2} A_{2}^{2} e^{4 \delta_{1}}+u_{0}^{2} A_{2}^{4} e^{6 \delta_{1}}\right] .
\end{aligned}
$$

This solution is nonsingular and bounded if $A_{2} e^{2 \delta_{1}}>1$ or $A_{2} e^{2 \delta_{1}}<-1$. When these conditions are relaxed the solution (76) may develop singularity in a finite time.

Example 7. Choose the parameters of the solution (76) as $\alpha_{1}=\frac{1}{2}, u_{0}=2, \delta_{1}=0, \alpha_{3}=5, a=4$. Therefore the solution (76) becomes

$$
|u(x, t)|^{2}=\frac{68449+45780 \sin \left(\frac{1}{2} x-\frac{23}{8} t\right)+12600 \sin \left(\frac{3}{2} x-\frac{69}{8} t\right)+39660 \cos \left(x-\frac{23}{4} t\right)+7200 \cos \left(2 x-\frac{23}{2} t\right)}{\left(60 \cos \left(x-\frac{23}{4} t\right)+109\right)^{2}} .
$$

The above solution is a nonsingular and bounded periodic wave solution. The graph of this solution is given in Fig. 7.

\section{Conclusion}

In this work, we have studied local and nonlocal integrable reductions of the symmetrical HS system. The local reductions yield KdV and complex KdV equations. The nonlocal reductions of the HS system give several type of new integrable nonlocal KdV equations. These are time (T)-, space (S)-, and space-time (ST)-reversal nonlocal complex KdV equations and space-time (ST)-reversal nonlocal KdV equation. By using the one-soliton solution of the HS system and the reduction formulas we obtained the one-soliton solutions of the reduced local and nonlocal KdV equations. Using our approach one can find also two- and three-soliton solutions of these KdV equations.

\section{CRediT authorship contribution statement}

Metin Gürses: Conceptualization, Methodology, Supervision, Conducting, Visualization, Writing-Review and Editing. Aslı Pekcan: Methodology, Validation, Formal Analysis, Writing-original draft, Writing-Review and Editing.

\section{Funding}

This research did not receive any specific grant from funding agencies in the public, commercial, or not-for-profit sectors.

\section{Declaration of competing interest}

The authors declare that they have no known competing financial interests or personal relationships that could have appeared to influence the work reported in this paper. 


\section{References}

[1] M.J. Ablowitz, Z.H. Musslimani, Integrable nonlocal nonlinear Schrödinger equation, Phys. Rev. Lett. 110 (2013) 064105.

[2] M.J. Ablowitz, Z.H. Musslimani, Inverse scattering transform for the integrable nonlocal nonlinear Schrödinger equation, Nonlinearity 29 (2016) $915-946$.

[3] M.J. Ablowitz, Z.H. Musslimani, Integrable nonlocal nonlinear equations, Stud. Appl. Math. 139 (1) (2016) 7-59.

[4] K. Chen, X. Deng, S. Lou, D. Zhang, Solutions of local and nonlocal equations reduced from the AKNS hierarchy, Stud. Appl. Math. 141 (1) (2018) 113-141.

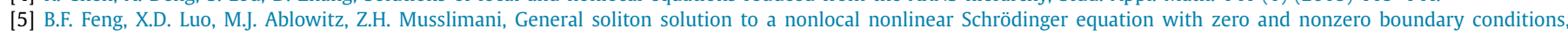
Nonlinearity 31 (12) (2018) 5385-5409.

[6] V.S. Gerdjikov, A. Saxena, Complete integrability of nonlocal nonlinear Schrödinger equation, J. Math. Phys. 58 (1) (2017) 013502.

[7] M. Gürses, A. Pekcan, Nonlocal nonlinear Schrödinger equations and their soliton solutions, J. Math. Phys. 59 (2018) 051501.

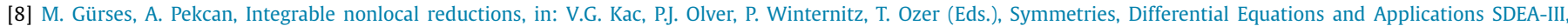
Istanbul, Turkey, August 2017, in: Springer Proceedings in Mathematics and Statistics, vol. 266, 2018, pp. 27-52.

[9] X. Huang, L. Ling, Soliton solutions for the nonlocal nonlinear Schrödinger equation, Eur. Phys. J. Plus 131 (2016) 148.

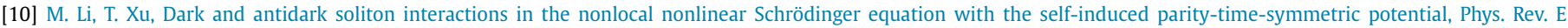
$91(2015) 033202$.

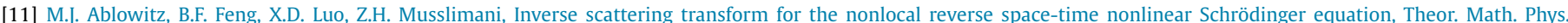
196 (3) (2018) 1241-1267.

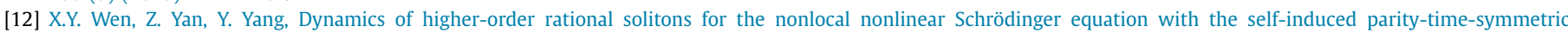
potential, Chaos 26 (2015) 063123.

[13] A. Khare, A. Saxena, Periodic and hyperbolic soliton solutions of a number of nonlocal nonlinear equations, J. Math. Phys. 56 (2015) 032104.

[14] J. Yang, General N-solitons and their dynamics in several nonlocal nonlinear Schrödinger equations, Phys. Lett. A 383 (4) (2019) $328-337$.

[15] M. Gürses, A. Pekcan, Nonlocal nonlinear modified KdV equations and their soliton solutions, Commun. Nonlinear Sci. Numer. Simul. 67 (2019) 427.

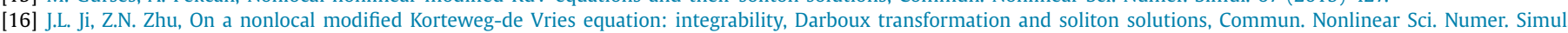
42 (2017) 699.

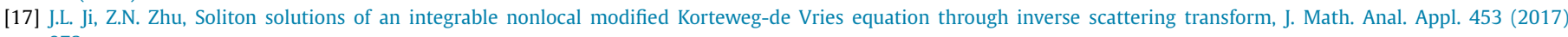
973.

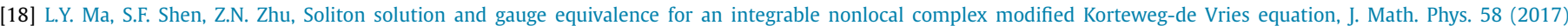
103501.

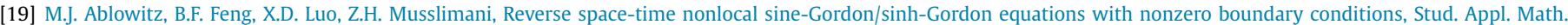
141 (3) (2018) 267-307.

[20] A.S. Fokas, Integrable multidimensional versions of the nonlocal Schrödinger equation, Nonlinearity 29 (2016) 319.

[21] J. Rao, Y. Zhang, A.S. Fokas, J. He, Rogue waves of the nonlocal Davey-Stewartson I equation, Nonlinearity 31 (2018) 4090-4107.

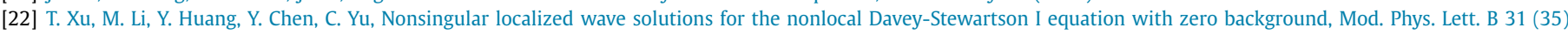
(2017) 1750338.

[23] Z.-X. Zhou, Darboux transformations global explicit solutions for nonlocal Davey-Stewartson I equation, Stud. Appl. Math. 141 (2) (2018) 186-204.

[24] Y. Zhang, Y. Liu, Breather and lump solutions for nonlocal Davey-Stewartson II equation, Nonlinear Dyn. 96 (2019) 107-113.

[25] M. Gürses, Nonlocal Fordy-Kulish equations on symmetric spaces, Phys. Lett. A 381 (2017) 1791.

[26] V.S. Gerdjikov, G.G. Grahovski, R.I. Ivanov, On the N-wave equations with PT symmetry, Theor. Math. Phys. 188 (3) (2016) 1305

[27] D. Sinha, P.K. Ghosh, Integrable nonlocal vector nonlinear Schrödinger equation with self-induced parity-time symmetric potential, Phys. Lett. A 381 (2017) 124.

[28] Z. Yan, Integrable PT-symmetric local and nonlocal vector nonlinear Schrödinger equations: a unified two parameter model, Appl. Math. Lett. 47 (2015) 61.

[29] V.S. Gerdjikov, G.G. Grahovski, R.I. Ivanov, On integrable wave interactions and Lax pairs on symmetric spaces, Wave Motion 71 (2017) 53.

[30] V.S. Gerdjikov, On nonlocal models of Kulish-Sklyanin type and generalized Fourier transforms, Stud. Comput. Intell. 681 (2017) 37.

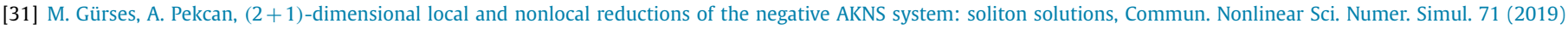
$161-173$.

[32] A. Pekcan, Nonlocal coupled HI-MKdV systems, Commun. Nonlinear Sci. Numer. Simul. 72 (2019) 493-515.

[33] M. Gürses, A. Pekcan, K. Zheltukhin, Nonlocal hydrodynamic type of equations, Commun. Nonlinear Sci. Numer. Simul. 85 (2020) 105242.

[34] M. Gürses, A. Pekcan, K. Zheltukhin, Discrete symmetries and nonlocal reductions, Phys. Lett. A 384 (2020) 120065.

[35] V. Caudrelier, Interplay between the inverse scattering method and Fokas's unified transform with an application, Stud. Appl. Math. 140 (2017) 3.

[36] B. Yang, J. Yang, Transformations between nonlocal and local integrable equations, Stud. Appl. Math. 140 (2017) 178.

[37] R. Hirota, J. Satsuma, Soliton solutions of the coupled Korteweg-de Vries equation, Phys. Lett. A 85 (8-9) (1981) 407-408

[38] R. Dodd, A. Fordy, On the integrability of a system of coupled KdV equations, Phys. Lett. A 89 (4) (1982) $168-170$.

[39] R.N. Aiyer, Recursion operator for coupled KdV equation, Phys. Lett. A 93 (8) (1983) 368-370.

[40] R.N. Aiyer, Factorization of the $2 \times 2$ matrix recursion operator of the coupled KdV equation, J. Math. Phys. 25 (1984) $3227-3230$

[41] W. Oevel, On the integrability of the Hirota-Satsuma system, Phys. Lett. A 94 (9) (1983) 404-407.

[42] D. Levi, A hierarchy of coupled Korteweg-de Vries equations, Phys. Lett. A 95 (1) (1983) 7-10.

[43] A. Ramani, B. Dorizzi, B. Grammaticos, Integrability of the Hirota-Satsuma equations: two tests, Phys. Lett. A 99 (9) (1983) $411-414$.

[44] P.K.H. Gragert, R. Martini, The explicit structure of the prolongation algebra of the Hirota-Satsuma system, Phys. Lett. A 101 (9) (1984) 469-472.

[45] M. Gürses, A. Karasu, Integrable coupled KdV systems, J. Math. Phys. 39 (4) (1998) 2103-2111.

[46] M. Gürses, A. Karasu, V. Sokolov, On the construction of recursion operator from Lax representation, J. Math. Phys. 39 (4) (1999) 6473-6490.

[47] H.W. Tam, W.X. Ma, X.B. Hu, D.L. Wang, The Hirota-Satsuma coupled KdV equation and a coupled Ito system revisited, J. Phys. Soc. Jpn. 69 (1) (2000) 45-52.

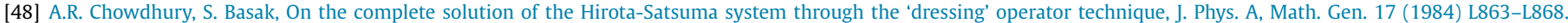

[49] H.C. Hu, Q.P. Liu, New Darboux transformation for Hirota-Satsuma coupled KdV system, Chaos Solitons Fractals 17 (2003) $921-928$.

[50] H.C. Hu, Y. Liu, New positon, negaton and complexiton solutions for the Hirota-Satsuma coupled KdV system, Phys. Lett. A 372 (2008) $5795-5798$.

[51] L. Sen-Yue, T. Xiao-Yan, L. Ji, Exact solutions of the coupled KdV system via a formally variable separation approach, Commun. Theor. Phys. 36 (2001) 145-148.

[52] J. Satsuma, R. Hirota, A coupled KdV equations is one case of the four-reduction of the KP hierarchy, J. Phys. Soc. Jpn. 51 (10) (1982) $3390-3397$.

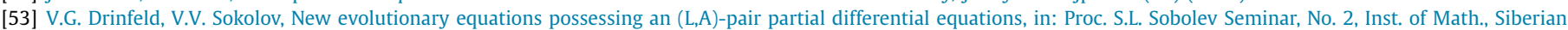
Branch of the USSR Acad. Sci., Novosibirsk, 1981 (in Russian).

[54] S.Yu. Sakovich, Coupled KdV equations of Hirota-Satsuma type, J. Nonlinear Math. Phys. 6 (3) (1999) 255-262.

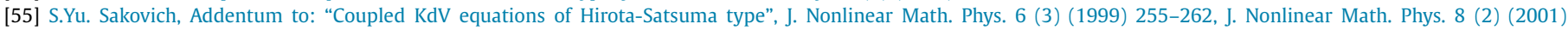
311-312.

[56] Z. Popowicz, The generalized Harry Dym equation, Phys. Lett. A 317 (3-4) (2003) 260-264.

[57] M.V. Foursov, Towards the complete classification of homogeneous two-component integrable equations, J. Math. Phys. 44 (7) (2003) $3088-3096$. 